\title{
Complex symmetric weighted composition operators on weighted Hardy space
}

\section{Opérateurs de composition pondérés symétriques complexes sur un espace de Hardy à poids}

\author{
Aastha Malhotra ${ }^{1}$ and Anuradha gupta ${ }^{2}$ \\ ${ }^{1}$ Faculty of Mathematical Sciences, Department of mathematics, University of Delhi, New Delhi-110007, India \\ aasthamalhotra01@gmail.com \\ ${ }^{2}$ Associate professor, Delhi college of arts and commerce, Department of mathematics, University of Delhi, Delhi, India \\ dishna2@yahoo.in
}

ABSTRACT. In this paper various conditions under which a weighted composition operator $W_{\psi, \phi}$ on the weighted Hardy space $H^{2}(\beta)$ becomes complex symmetric with respect to some special conjugation have been explored. We also investigate some important properties of the complex symmetric operator $W_{\psi, \phi}$, such as hermiticity and isometry.

2010 Mathematics Subject Classification. 47B33, 47B38.

KEYWORDS. Composition operator, Complex symmetric, Weighted Hardy space, Conjugation.

\section{Introduction and Preliminaries}

Let $\mathbb{D}=\{z \in \mathbb{C}:|z|<1\}$ be the open unit disk and $\overline{\mathbb{D}}$ denote the closure of the open unit disk $\mathbb{D}$. Let $H(\mathbb{D})$ denote the space of all analytic functions on $\mathbb{D}$. Then for any given self-analytic map $\phi$ on $\mathbb{D}$, the composition operator on $H(\mathbb{D})$ is defined by $C_{\phi} f=f \circ \phi$ for $f \in H(\mathbb{D})$ where $\phi$ is known as the symbol of the composition operator $C_{\phi}$. The restriction of the composition operator $C_{\phi}$ to various Banach spaces has been studied extensively in the last several decades and $[1,10]$ are good sources for studying the basic theory of composition operators on various function spaces.

A Hilbert space $\mathcal{H}$ whose vectors are functions analytic on the unit disk $\mathbb{D}$ is called a weighted Hardy space [1, Page 14] if the monomials $1, z, z^{2}, \ldots$ constitute an orthogonal set of nonzero vectors in the Hilbert space $\mathcal{H}$ and the polynomials are also dense in $\mathcal{H}$. Let $\|\cdot\|$ denote the norm on the Hilbert space $\mathcal{H}$. Consider a sequence $\{\beta(n)\}$ with $\beta(0)=\|1\|=1$ and $\beta(n)=\left\|z^{n}\right\|$ for every $n \in \mathbb{N}$. Such a sequence is called a weight sequence. Each of the weighted Hardy space is characterized by such a weight sequence $\{\beta(n)\}$. For a given weight sequence $\{\beta(n)\}$, the associated weighted Hardy space is denoted by $H^{2}(\beta)$ and is defined as:

$$
H^{2}(\beta)=\left\{f(z)=\sum_{n=0}^{\infty} a_{n} z^{n}: \sum_{n=0}^{\infty}\left|a_{n}\right|^{2} \beta(n)^{2}<\infty\right\}
$$

and the inner product on $H^{2}(\beta)$ is given by:

$$
\left\langle\sum_{n=0}^{\infty} a_{n} z^{n}, \sum_{n=0}^{\infty} b_{n} z^{n}\right\rangle=\sum_{n=0}^{\infty} a_{n} \overline{b_{n}} \beta(n)^{2} .
$$

Every function in the weighted Hardy space $H^{2}(\beta)$ is analytic in $\mathbb{D}$. The Hardy space, the Bergman space, the Dirichlet space and the $S^{2}(\mathbb{D})$ space are the weighted Hardy spaces which are identified with the weight sequences $\beta(n)=1, \beta(n)=(n+1)^{-1 / 2}, \beta(n)=n^{1 / 2}$ and $\beta(n)=n$, respectively. The 
function $k(z)=\sum_{n=0}^{\infty} \frac{z^{n}}{\beta(n)^{2}}$ is called the generating function for the weighted Hardy space $H^{2}(\beta)$. This generating function for $H^{2}(\beta)$ is analytic on $\mathbb{D}$. For each point $w$ in the open unit disk $\mathbb{D}$, evaluation of functions in $H^{2}(\beta)$ at $w$ is a bounded linear functional and for all $f$ in $H^{2}(\beta), f(w)=\left\langle f, K_{w}\right\rangle$ where $K_{w}(z)=k(\bar{w} z)=\sum_{n=0}^{\infty} \frac{(\bar{w} z)^{n}}{\beta(n)^{2}}$ [1, Theorem 2.10]. The function $K_{w}$ is called the reproducing kernel of the Hilbert space $H^{2}(\beta)$.

On a complex Hilbert space $\mathcal{H}$, a conjugation $\mathcal{C}: \mathcal{H} \rightarrow \mathcal{H}$ is a function which satisfies the following:

1. $\mathcal{C}$ is an antilinear mapping: $\mathcal{C}(\alpha x+\beta y)=\bar{\alpha} \mathcal{C}(x)+\bar{\beta} \mathcal{C}(y)$ for every $x, y$ in $\mathcal{H}$ and for all $\alpha, \beta$ in $\mathbb{C}$.

2. $\mathcal{C}$ is an involution: $\mathcal{C}^{2}=I$ where $I$ is an identity operator on $\mathcal{H}$.

3. $\mathcal{C}$ is an isometry: $\|\mathcal{C} x\|=\|x\|$ for every $x$ in $\mathcal{H}$.

The mapping $\mathcal{J}$, which is defined as $(\mathcal{J} f)(z)=\overline{f(\bar{z})}$ where $f \in H(\mathbb{D})$, is a conjugation and is known as the standard conjugation. In this paper, we consider the following generalization of the conjugation function

$$
\mathcal{J}_{\mu}: H^{2}(\beta) \rightarrow H^{2}(\beta)
$$

which is defined as

$$
\left(\mathcal{J}_{\mu} f\right)(z)=\overline{f(\mu \bar{z})}, \quad \forall z \in \mathbb{D}
$$

where $\mu \in \mathbb{C}$ is such that $|\mu|=1$ and $f \in H^{2}(\beta)$. Suppose that $\mathcal{C}$ is a conjugation on a Hilbert space $\mathcal{H}$, then a bounded linear operator $T$ is said to be $\mathcal{C}$-symmetric on $\mathcal{H}$ if $T=\mathcal{C} T^{*} \mathcal{C}$. An operator $T$ is said to be complex symmetric if there exists a conjugation $\mathcal{C}$ with respect to which $T$ is $\mathcal{C}$-symmetric. Moreover, it is shown in [3] that for a complex symmetric operator $T$ on a Hilbert space $\mathcal{H}$, we can find an orthonormal basis $\left\{e_{n}\right\}_{n=0}^{\infty}$ of $\mathcal{H}$ with respect to which the matrix representation of $T$ is complex symmetric (i.e. Thas a self-transpose matrix representation with respect to $\left\{e_{n}\right\}_{n=0}^{\infty}$ ). Garcia, Putinar and Wogen $[4,5,6]$ initiated the general study of the complex symmetric operators during the first decade of twenty first century. Examples of complex symmetric operators are quite diverse which includes all the normal operators, compressed Toeplitz operators, Hankel operators, Volterra integral operators etc. Many authors have studied the complex symmetric structure of the weighted composition operators on several reproducing kernel Hilbert spaces such as on the Hardy space $H^{2}(\mathbb{D})$ [7, 9], on the space $H_{\gamma}(\mathbb{D})[8]$ in which the reproducing kernels for the evaluation of the functions are of the form $K_{w}^{\gamma}(z)=$ $\frac{1}{(1-\bar{w} z)^{\gamma}}, \gamma \in \mathbb{N}$. Garcia and Hammond [2] identified several new classes of the complex symmetric weighted composition operators on the weighted Hardy space $H^{2}(\beta)$. In this article, we aim to find the characterizations of the symbols $\phi$ and $\psi$ so that the weighted composition operator $W_{\psi, \phi}$ is complex symmetric on $H^{2}(\beta)$ with respect to the conjugation $\mathcal{J}_{\mu}$. In the last section, we also find the conditions for hermiticity and isometry for the $\mathcal{J}_{\mu}$-symmetric operator $W_{\psi, \phi}$.

\section{Complex symmetry on weighted Hardy space}

Let $\psi: \mathbb{D} \rightarrow \mathbb{C}$ and $\phi: \mathbb{D} \rightarrow \mathbb{D}$ be two analytic maps. Then the weighted composition operator $W_{\psi, \phi}$ on $H^{2}(\beta)$ is defined as $\left(W_{\psi, \phi} f\right)(z)=\psi(z) \cdot(f \circ \phi)(z)$ for $f \in H^{2}(\beta)$ and $z \in \mathbb{D}$. In the following theorem, we find the structure of the symbols $\phi$ and $\psi$ so that the weighted composition operator $W_{\psi, \phi}$ is complex symmetric with respect to the conjugation $\mathcal{J}_{\mu}$ on the weighted Hardy space $H^{2}(\beta)$. 
Theorem 2.1. Let $W_{\psi, \phi}$ be a bounded complex symmetric weighted composition operator with respect to the conjugation $\mathcal{J}_{\mu}$ on $H^{2}(\beta)$. Then

$$
\phi(z)=a_{0}+\beta(1)^{2} a_{1} z \frac{q(z)}{p(z)}
$$

and

$$
\psi(z)=a_{2} p(z)
$$

where $a_{0}=\phi(0), a_{1}=\phi^{\prime}(0), a_{2}=\psi(0), p(z)=\sum_{n=0}^{\infty} \frac{\left(\bar{\mu} a_{0} z\right)^{n}}{\beta(n)^{2}}$ and $q(z)=\sum_{n=1}^{\infty} \frac{n\left(\bar{\mu} a_{0} z\right)^{n-1}}{\beta(n)^{2}}$.

Proof. Let $W_{\psi, \phi}$ be complex symmetric with conjugation $\mathcal{J}_{\mu}$. Let $w$ and $z$ be the points in the open unit disk $\mathbb{D}$. Then $\left(W_{\psi, \phi} \mathcal{J}_{\mu}\right) K_{w}(z)=\left(\mathcal{J}_{\mu} W_{\psi, \phi}^{*}\right) K_{w}(z)$ if and only if $\psi(z) K_{\mu \bar{w}}(\phi(z))=\psi(w) K_{\mu \overline{\phi(w)}}(z)$ if and only if

$$
\psi(z) \sum_{n=0}^{\infty} \frac{(\bar{\mu} w \phi(z))^{n}}{\beta(n)^{2}}=\psi(w) \sum_{n=0}^{\infty} \frac{(\bar{\mu} \phi(w) z)^{n}}{\beta(n)^{2}} .
$$

On putting $w=0$ in (2.3), we get

$$
\psi(z) \cdot \frac{1}{\beta(0)^{2}}=\psi(0) \cdot \sum_{n=0}^{\infty} \frac{(\bar{\mu} \phi(0) z)^{n}}{\beta(n)^{2}} .
$$

Let $a_{0}=\phi(0), a_{2}=\psi(0)$ and $p(z)=\sum_{n=0}^{\infty} \frac{\left(\bar{\mu} a_{0} z\right)^{n}}{\beta(n)^{2}}$. Then $\psi(z)=a_{2} p(z)$.

Substituting $\psi(z)$ in (2.3), we obtain

$$
a_{2} p(z)\left(\sum_{n=0}^{\infty} \frac{(\bar{\mu} w \phi(z))^{n}}{\beta(n)^{2}}\right)=a_{2} p(w)\left(\sum_{n=0}^{\infty} \frac{(\bar{\mu} \phi(w) z)^{n}}{\beta(n)^{2}}\right) .
$$

Differentiating the last expression with respect to $w$, we get

$$
\begin{aligned}
p(z)\left(\sum_{n=1}^{\infty} \frac{n(\bar{\mu} w \phi(z))^{n-1} \bar{\mu} \phi(z)}{\beta(n)^{2}}\right)= & p(w)\left(\sum_{n=1}^{\infty} \frac{n(\bar{\mu} \phi(w) z)^{n-1} \bar{\mu} z \phi^{\prime}(w)}{\beta(n)^{2}}\right)+ \\
& \left(\sum_{n=1}^{\infty} \frac{n\left(\bar{\mu} a_{0} w\right)^{n-1} \bar{\mu} a_{0}}{\beta(n)^{2}}\right)\left(\sum_{n=0}^{\infty} \frac{(\bar{\mu} \phi(w) z)^{n}}{\beta(n)^{2}}\right) .
\end{aligned}
$$

Putting $w=0$ in (2.4), we get

$$
p(z) \frac{\bar{\mu} \phi(z)}{\beta(1)^{2}}=\left(\sum_{n=1}^{\infty} \frac{n\left(\bar{\mu} a_{0} z\right)^{n-1} \bar{\mu} z \phi^{\prime}(0)}{\beta(n)^{2}}\right)+\frac{\bar{\mu} a_{0}}{\beta(1)^{2}} p(z) .
$$

Let $a_{1}=\phi^{\prime}(0)$ and $q(z)=\sum_{n=1}^{\infty} \frac{n\left(\bar{\mu} a_{0} z\right)^{n-1}}{\beta(n)^{2}}$. Then,

$$
\frac{p(z) \bar{\mu} \phi(z)}{\beta(1)^{2}}=\frac{\bar{\mu} a_{0}}{\beta(1)^{2}} p(z)+\bar{\mu} a_{1} z q(z) .
$$

Hence, $\phi(z)=a_{0}+\beta(1)^{2} a_{1} z \frac{q(z)}{p(z)}$ and $\psi(z)=a_{2} p(z)$ for every $z \in \mathbb{D}$. 
Corollary 2.2. For a bounded weighted composition operator $W_{\psi, \phi}$ on $H^{2}(\beta)$, let the analytic functions $\phi: \mathbb{D} \rightarrow \mathbb{D}$ and $\psi: \mathbb{D} \rightarrow \mathbb{C}$ be of the form

$$
\phi(z)=a_{0}+\beta(1)^{2} a_{1} z \frac{q(z)}{p(z)}
$$

and $\psi(z)=a_{2} p(z)$ where $a_{0}=\phi(0), a_{1}=\phi^{\prime}(0), a_{2}=\psi(0), p(z)=\sum_{n=0}^{\infty} \frac{\left(\bar{\mu} a_{0} z\right)^{n}}{\beta(n)^{2}}$ and $q(z)=$ $\sum_{n=1}^{\infty} \frac{n\left(\bar{\mu} a_{0} z\right)^{n-1}}{\beta(n)^{2}}$ for every $z \in \mathbb{D}$. If either of $a_{0}$ or $a_{1}$ is zero, then the operator $W_{\psi, \phi}$ is complex symmetric with respect to the conjugation $\mathcal{J}_{\mu}$ on $H^{2}(\beta)$.

Proof. It can be easily observed that if both $a_{0}$ and $a_{1}$ are zero, then $W_{\psi, \phi}$ is complex symmetric with respect to the conjugation $\mathcal{J}_{\mu}$. Next, we consider the following two cases:

Case (i) : If $a_{0}$ is zero and $a_{1}$ is non-zero, then $p(z)=1$ and $q(z)=\frac{1}{\beta(1)^{2}}$. Thus, we get that $\phi(z)=a_{1} z$ and $\psi(z)=a_{2}$. On substituting these expressions for $\phi(z)$ and $\psi(z)$ in (2.3), we obtain that $W_{\psi, \phi}$ is complex symmetric with respect to the conjugation $\mathcal{J}_{\mu}$.

Case (ii) : If $a_{0}$ is non-zero and $a_{1}$ is zero, then $\phi(z)=a_{0}$ and $\psi(z)=a_{2} p(z)$. Hence, we can see that the equation (2.3) is satisfied which proves that $W_{\psi, \phi}$ is complex symmetric with respect to the conjugation $\mathcal{J}_{\mu}$.

For the Hardy space $H^{2}(\mathbb{D})$, Jung et al. in [7] provided the structure of the symbols $\phi$ and $\psi$ for a $\mathcal{J}$-symmetric weighted composition operator $W_{\psi, \phi}$ where $\mathcal{J}=\mathcal{J}_{\mu}$ for $\mu=1$. This result is stated below:

Theorem 2.3 ([7]). Let $\phi$ be an analytic selfmap of $\mathbb{D}$ and let $\psi \in H^{\infty}(\mathbb{D})$ be not identically zero. If the weighted composition operator $W_{\psi, \phi}$ is complex symmetric with conjugation $\mathcal{J}$ on $H^{2}(\mathbb{D})$ where $(\mathcal{J} f)(z)=\overline{f(\bar{z})}$, then $\phi(z)=a_{0}+\frac{a_{1} z}{1-a_{0} z}$ and $\psi(z)=\frac{a_{2}}{1-a_{0} z}$ where $a_{0}=\phi(0), a_{1}=\phi^{\prime}(0)$ and $a_{2}=\psi(0)$.

We illustrate with the help of following example that how the above theorem for the Hardy space can be obtained from Theorem 2.1:

Example: The Hardy space $H^{2}(\mathbb{D})$ is identified by the weight sequence $<\beta(n)>=<1>$. Thus, on substituting $\beta(n)=1=\mu$ in (2.1) and (2.2), we obtain

$$
\begin{aligned}
\phi(z)= & a_{0}+a_{1} z \frac{\sum_{n=1}^{\infty} n\left(a_{0} z\right)^{n-1}}{\sum_{n=0}^{\infty}\left(a_{0} z\right)^{n}} \\
= & a_{0}+a_{1} z\left(\frac{1+2 a_{0} z+3\left(a_{0} z\right)^{2}+4\left(a_{0} z\right)^{3}+\cdots}{\sum_{n=0}^{\infty}\left(a_{0} z\right)^{n}}\right) \\
= & a_{0}+a_{1} z\left(\frac{1+a_{0} z+\left(a_{0} z\right)^{2}+\left(a_{0} z\right)^{3}+\cdots}{\sum_{n=0}^{\infty}\left(a_{0} z\right)^{n}}+\frac{a_{0} z+\left(a_{0} z\right)^{2}+\left(a_{0} z\right)^{3}+\cdots}{\sum_{n=0}^{\infty}\left(a_{0} z\right)^{n}}\right. \\
& \left.+\frac{\left(a_{0} z\right)^{2}+\left(a_{0} z\right)^{3}+\cdots}{\sum_{n=0}^{\infty}\left(a_{0} z\right)^{n}}+\cdots\right) \\
= & a_{0}+a_{1} z \sum_{n=0}^{\infty}\left(a_{0} z\right)^{n}\left(\text { since, we have }\left|a_{0} z\right|<1\right) \\
= & a_{0}+\frac{a_{1} z}{1-a_{0} z}
\end{aligned}
$$

and $\psi(z)=a_{2} p(z)=a_{2} \sum_{n=0}^{\infty}\left(a_{0} z\right)^{n}=\frac{a_{2}}{1-a_{0} z}$ for every $z \in \mathbb{D}$. 
Next, we find the conditions for a bounded composition operator $C_{\phi}$ on $H^{2}(\beta)$ to be complex symmetric with respect to the conjugation $\mathcal{J}_{\mu}$.

Corollary 2.4. A bounded composition operator $C_{\phi}$ on $H^{2}(\beta)$ is complex symmetric with respect to the conjugation $\mathcal{J}_{\mu}$ if and only if for all $z \in \mathbb{D}, \phi(z)=$ az where $a \in \overline{\mathbb{D}}$.

Proof. Taking $\psi \equiv 1$ in Theorem 2.1, we get $\psi(0)=a_{2}=1$ and $p(z)=1$ for all $z \in \mathbb{D}$. Hence, if $C_{\phi}$ is complex symmetric on $H^{2}(\beta)$ with respect to the conjugation $\mathcal{J}_{\mu}$ then $\phi(z)=a_{1} z$ where $a_{1}=\phi^{\prime}(0)$.

Conversely, if $\phi(z)=a z$ is a self-analytic map on $\mathbb{D}$ then it can be seen directly using simple calculations that

$$
C_{\phi} \mathcal{J}_{\mu} K_{w}(z)=\sum_{n=0}^{\infty} \frac{(a \bar{\mu} w z)^{n}}{\beta(n)^{2}}=\mathcal{J}_{\mu} C_{\phi}^{*} K_{w}(z)
$$

for all $z, w \in \mathbb{D}$. Hence, $C_{\phi}$ is complex symmetric with conjugation $\mathcal{J}_{\mu}$.

Lemma 2.5 ([6]). If $T^{2}$ is normal, then $T$ is a complex symmetric operator.

In the next result, we deduce the conditions under which the converse of Lemma 2.5 holds for a weighted composition operator on the weighted Hardy space.

Theorem 2.6. Let $W_{\psi, \phi}$ be a bounded operator on $H^{2}(\beta)$ such that $\phi(0)=0$. If $W_{\psi, \phi}$ is $\mathcal{J}_{\mu}$-symmetric, then $W_{\psi, \phi}^{2}$ is a normal operator.

Proof. Suppose that $W_{\psi, \phi}$ is $\mathcal{J}_{\mu}$-symmetric where $\phi(0)=0$. Then $\psi(z)=a_{2}$ and $\phi(z)=a_{1} z$ where $a_{1}=\phi^{\prime}(0)$ and $a_{2}=\psi(0)$. Hence, we obtain that $W_{\psi, \phi}^{2}$ is a normal operator as $W_{\psi, \phi}^{2}\left(W_{\psi, \phi}^{2}\right)^{*} K_{w}(z)=$ $\left|a_{2}\right|^{4} K_{\left|a_{1}\right|{ }^{4} w}(z)=\left(W_{\psi, \phi}^{2}\right)^{*} W_{\psi, \phi}^{2} K_{w}(z)$ for every $w, z \in \mathbb{D}$.

We say that an analytic function $\phi: \mathbb{D} \rightarrow \mathbb{D}$ is an automorphism on $\mathbb{D}$ if it is bijective. It is well known that the automorphisms of the unit disk are the functions of the form

$$
\phi(z)=k \frac{c-z}{1-\bar{c} z}
$$

where $k \in \mathbb{C},|k|=1$ and $|c|<1$.

In the next result, we explore the condition on $\phi$ so that it is an automorphism on $\mathbb{D}$ and simultaneously satisfies (2.1).

Proposition 2.7. Let $W_{\psi, \phi}$ be bounded and complex symmetric with respect to conjugation $\mathcal{J}_{\mu}$ on $H^{2}(\beta)$. If $\phi$ is an automorphism on $\mathbb{D}$, then either one of the following holds:

1. there exists $k \in \mathbb{C},|k|=1$ such that

$$
\phi(z)=-k z
$$

where $a_{0}=0$ and $a_{1}=-k$.

2. there exist $c \in \mathbb{D}, c \neq 0$ and $k \in \mathbb{C}$ with $|k|=1$ such that

$$
\phi(z)=k \frac{c-z}{1-\bar{c} z}
$$

where $a_{0}=k c, a_{1}=\frac{\bar{\mu} c k^{2}\left(|c|^{2}-1\right) \beta(2)^{2}}{\beta(1)^{2}\left(2 \beta(1)^{2} \bar{\mu} c k-\bar{c} \beta(2)^{2}\right)}$ and $k=\frac{\bar{c} \beta(1)^{2} \beta(2)^{2}}{c \bar{\mu}\left(2 \beta(1)^{4}-\beta(2)^{2}\right)}$. 
Proof. Since $W_{\psi, \phi}$ is $\mathcal{J}_{\mu}$-symmetric on $H^{2}(\beta)$, Theorem 2.1 gives that

$$
\phi(z)=a_{0}+\beta(1)^{2} a_{1} z \frac{q(z)}{p(z)}
$$

where $a_{0}=\phi(0), a_{1}=\phi^{\prime}(0), a_{2}=\psi(0), p(z)=\sum_{n=0}^{\infty} \frac{\left(\bar{\mu} a_{0} z\right)^{n}}{\beta(n)^{2}}$ and $q(z)=\sum_{n=1}^{\infty} \frac{n\left(\bar{\mu} a_{0} z\right)^{n-1}}{\beta(n)^{2}}$ for every $z \in \mathbb{D}$. If $\phi$ is an automorphism on $\mathbb{D}$, then there exist complex numbers $c$ and $k$ where $|c|<1$ and $|k|=1$ such that for all $z \in \mathbb{D}$, we have $\phi$ of the form

$$
\phi(z)=\frac{k c-k z}{1-\bar{c} z} .
$$

For an analytic function $\phi$ given by (2.5) to be of the form given in (2.6), we equate (2.5) and (2.6) which gives

$$
a_{0}+\beta(1)^{2} a_{1} z \frac{q(z)}{p(z)}=\frac{k c-k z}{1-\bar{c} z}
$$

or, equivalently we have

$$
a_{0} p(z)-a_{0} \bar{c} z p(z)+\beta(1)^{2} a_{1} z q(z)-\beta(1)^{2} \bar{c} a_{1} z^{2} q(z)=k c p(z)-k z p(z) .
$$

Comparing the constants on both the sides, we get

$$
a_{0}=c k \text {. }
$$

Again, comparing the coefficients of $z$ and $z^{2}$ respectively, we obtain

$$
\frac{\bar{\mu} a_{0}^{2}}{\beta(1)^{2}}-a_{0} \bar{c}+a_{1}=\frac{\bar{\mu} a_{0} c k}{\beta(1)^{2}}-k
$$

and

$$
\frac{\bar{\mu}^{2} a_{0}^{3}}{\beta(2)^{2}}-\frac{\bar{\mu} a_{0}^{2} \bar{c}}{\beta(1)^{2}}+\frac{2 \bar{\mu} \beta(1)^{2} a_{0} a_{1}}{\beta(2)^{2}}-a_{1} \bar{c}=\frac{\bar{\mu}^{2} a_{0}^{2} c k}{\beta(2)^{2}}-\frac{\bar{\mu} a_{0} k}{\beta(1)^{2}} .
$$

If $c=0$, then (2.7) gives $a_{0}=0$. Putting $a_{0}=0$ in (2.8), we obtain $a_{1}=-k$. Substituting $a_{0}=0$ in the expressions for $q(z)$ and $p(z)$, we get $q(z)=\frac{1}{\beta(1)^{2}}$ and $p(z)=1$ for all $z \in \mathbb{D}$. Thus, we have $\phi(z)=-k z$ where $|-k|=1$.

Let $c \in \mathbb{D}$ and $c \neq 0$. Putting $a_{0}=c k$ in (2.9), we get

$$
a_{1}=\frac{\bar{\mu} c k^{2}\left(|c|^{2}-1\right) \beta(2)^{2}}{\beta(1)^{2}\left(2 \beta(1)^{2} \bar{\mu} c k-\bar{c} \beta(2)^{2}\right)} .
$$

Further substituting $a_{0}$ and $a_{1}$ in (2.8), we obtain

$$
k\left(|c|^{2}-1\right)-\frac{\bar{\mu} c k^{2}\left(|c|^{2}-1\right) \beta(2)^{2}}{\beta(1)^{2}\left(2 \beta(1)^{2} \bar{\mu} c k-\bar{c} \beta(2)^{2}\right)}=0 .
$$

Since $|c|<1$, we get that $1-|c|^{2} \neq 0$. Also, $k \neq 0$ as $|k|=1$. Therefore, we have

$$
k=\frac{\bar{c} \beta(1)^{2} \beta(2)^{2}}{c \bar{\mu}\left(2 \beta(1)^{4}-\beta(2)^{2}\right)} .
$$

Hence, $\phi(z)=k \frac{c-z}{1-\bar{c} z}$ where $k$ is given by (2.10). 
An operator $T \in B(\mathcal{H})$ is said to be idempotent if $T^{2}=T$. We obtain the structure of the symbols $\phi$ and $\psi$ for an idempotent weighted composition operator $W_{\psi, \phi}$ on $H^{2}(\beta)$.

Theorem 2.8. Let $W_{\psi, \phi}: H^{2}(\beta) \rightarrow H^{2}(\beta)$ be a weighted composition operator where $\psi: \mathbb{D} \rightarrow \mathbb{C}$ is an analytic map and $\phi: \mathbb{D} \rightarrow \mathbb{D}$ is a non-constant self-analytic map. If $W_{\psi, \phi}$ is an idempotent operator, then $\psi(z)=1$ for all $z \in \mathbb{D}$ (i.e $\psi \equiv 1$ ).

Proof. Suppose that $W_{\psi, \phi}$ is an idempotent operator. Then

$$
W_{\psi, \phi}^{2} K_{w}(z)=W_{\psi, \phi} K_{w}(z)
$$

if and only if

$$
\psi(\phi(z)) K_{w}\left(\phi^{2}(z)\right)=K_{w}(\phi(z))
$$

for every $z, w \in \mathbb{D}$. On putting $w=0$ in (2.11), we get that $(\psi \circ \phi)(z)=1$ for every $z \in \mathbb{D}$. Since $\phi$ is a non-constant self-analytic map on $\mathbb{D}$, the image of the unit disk $\mathbb{D}$ under $\phi($ i.e, $\phi(\mathbb{D}))$ is an open and connected set in $\mathbb{D}$. As $\psi(z)=1$ for every $z \in \phi(\mathbb{D})$, on using the identity theorem we get that $\psi(z)=1$ for every $z \in \mathbb{D}$.

Remark 2.9. The converse of the above theorem is not true in general. For example, if we consider $\phi(z)=-z$ then $\phi$ is a non-constant self-analytic map on $\mathbb{D}$ but (2.11) does not hold as $K_{w}\left(\phi^{2}(z)\right)=$ $K_{w}(z) \neq K_{w}(-z)=K_{w}(\phi(z))$ for every $z, w \in \mathbb{D}$. Hence, $W_{\psi, \phi}$ is not idempotent.

But the converse holds if we consider $\phi$ to be an idempotent self-analytic function on $\mathbb{D}$, then (2.11) holds which implies that $W_{\psi, \phi}$ is idempotent.

It can also be noted that if we consider $\phi$ to be a constant map on $\mathbb{D}$ i.e. $\phi(z)=c$ for every $z \in \mathbb{D}$ where $c \in \mathbb{D}$ and take $\psi: \mathbb{D} \rightarrow \mathbb{C}$ to be analytic, then it follows from (2.11) that the weighted composition operator $W_{\psi, \phi}$ is an idempotent operator if and only if $\psi(c)=1$.

Proposition 2.10. Let $\phi$ be an automorphism on $\mathbb{D}$. Then $\phi^{2}(z)=\phi(z)$ for every $z \in \mathbb{D}$ if and only if $\phi(z)=z$ for every $z \in \mathbb{D}$.

The following lemma provides condition under which a bounded linear operator on a separable complex Hilbert space $\mathcal{H}$ becomes complex symmetric.

Lemma 2.11 ([6]). If $T \in B(\mathcal{H})$ is algebraic of degree less than or equal to two, then $T$ is a complex symmetric operator.

As a consequence of the previous lemma, we have that if $T$ is idempotent (i.e. $T^{2}=T$ ) or nilpotent of order 2 (i.e. $T^{2}=0$ ), then the operator $T$ is complex symmetric.

Corollary 2.12. Let $W_{\psi, \phi}$ be a bounded weighted composition operator on $H^{2}(\beta)$. If the symbols $\phi$ and $\psi$ satisfy either of the following:

1. $\phi$ is an idempotent self-analytic map on $\mathbb{D}$ and $\psi \equiv 1$,

2. $\phi$ is a constant function, say $c($ where $|c|<1)$ and $\psi$ is any analytic map on $\mathbb{D}$ with $\psi(c)=1$,

then $W_{\psi, \phi}$ is a complex symmetric operator on $H^{2}(\beta)$. 
Proof. It follows from both the cases that $W_{\psi, \phi}$ is an idempotent operator and hence, $W_{\psi, \phi}$ is complex symmetric.

\section{Properties of complex symmetric operator $W_{\psi, \phi}$}

Recall that a bounded linear operator $T$ on $\mathcal{H}$ is said to be Hermitian if $T=T^{*}$. If a bounded linear operator $T$ is Hermitian, then it is complex symmetric on $\mathcal{H}$. In the following theorem, we determine the neecessary and sufficient conditions under which a bounded and $\mathcal{J}_{\mu}$-symmetric weighted composition operator becomes Hermitian on $H^{2}(\beta)$.

Theorem 3.1. Let the weighted composition operator $W_{\psi, \phi}$ be bounded and complex symmetric with respect to the conjugation $\mathcal{J}_{\mu}$ where the symbols $\phi$ and $\psi$ are as in (2.1) and (2.2). Then $W_{\psi, \phi}$ is Hermitian if and only if $a_{1}, a_{2} \in \mathbb{R}$ and $\bar{\mu} a_{0}=\overline{a_{0}}$ where $a_{0}=\phi(0), a_{1}=\phi^{\prime}(0)$ and $a_{2}=\psi(0)$.

Proof. Since $W_{\psi, \phi}$ is complex symmetric with respect to the conjugation $\mathcal{J}_{\mu}$, we have $\mathcal{J}_{\mu} W_{\psi, \phi}=$ $W_{\psi, \phi}^{*} \mathcal{J}_{\mu}$. We can observe that $W_{\psi, \phi}=W_{\psi, \phi}^{*}$ if and only if $W_{\psi, \phi} \mathcal{J}_{\mu}=W_{\psi, \phi}^{*} \mathcal{J}_{\mu}=\mathcal{J}_{\mu} W_{\psi, \phi}$. Let us suppose that $W_{\psi, \phi}$ is Hermitian. Then, for $z, w \in \mathbb{D}$, we have

$$
W_{\psi, \phi} \mathcal{J}_{\mu} K_{w}(z)=\mathcal{J}_{\mu} W_{\psi, \phi} K_{w}(z)
$$

which yields

$$
\psi(z) K_{\mu \bar{w}}(\phi(z))=\overline{\psi(\mu \bar{z})} K_{\bar{w}}(\overline{\phi(\mu \bar{z})})
$$

On putting $w=0$ in (3.1), we obtain that for every $z \in \mathbb{D}$

$$
\psi(z)=\overline{\psi(\mu \bar{z})}
$$

which implies that

$$
a_{2} \sum_{n=0}^{\infty} \frac{\left(\bar{\mu} a_{0} z\right)^{n}}{\beta(n)^{2}}=\overline{a_{2}} \sum_{n=0}^{\infty} \frac{\left(\overline{a_{0}} z\right)^{n}}{\beta(n)^{2}} .
$$

On putting $z=0$ in (3.3), we get that $a_{2}=\overline{a_{2}}$. It can be noted that on differentiating (3.3) on the both sides with respect to $z$ and then substituting $z=0$, we have $\frac{a_{2} \bar{\mu} a_{0}}{\beta(1)^{2}}=\frac{\overline{a_{2}} \overline{a_{0}}}{\beta(1)^{2}}$ which further implies that $\bar{\mu} a_{0}=\overline{a_{0}}$.

For $z \in \mathbb{D}$, write $\frac{q(z)}{p(z)}=\sum_{m=0}^{\infty} c_{m} z^{m}$ where $c_{m} \in \mathbb{C}$ for every $m \in \mathbb{N} \cup\{0\}$. Now, from (3.1) and (3.2) we observe that $K_{\mu \bar{w}}(\phi(z))=K_{\bar{w}}(\overline{\phi(\mu \bar{z})})$ for all $z, w \in \mathbb{D}$. This implies that for every $z, w \in \mathbb{D}$,

$$
\sum_{n=0}^{\infty} \frac{(\bar{\mu} w \phi(z))^{n}}{\beta(n)^{2}}=\sum_{n=0}^{\infty} \frac{(w \overline{\phi(\mu \bar{z})})^{n}}{\beta(n)^{2}}
$$

First, on differentiating (3.4) on the both sides with respect to $z$ and putting $z=0$ and then differentiating again the resulting expressions on the both sides with respect to $w$ and on putting $w=0$, we get $\bar{\mu} a_{1} c_{0}=$ $\bar{\mu} \overline{a_{1}} \overline{c_{0}}$. Since $c_{0}=q(0) / p(0)=1 / \beta(1)^{2}$ is real, we conclude that $a_{1}=\overline{a_{1}}$.

Conversely, let us suppose that $a_{1}, a_{2} \in \mathbb{R}$ and $\bar{\mu} a_{0}=\overline{a_{0}}$. Then in order to show that $W_{\psi, \phi}$ is Hermitian, it is sufficient to prove that (3.1) holds for every $z, w \in \mathbb{D}$. It can be noted that $p(z)=\sum_{n=0}^{\infty} \frac{\left(\bar{\mu} a_{0} z\right)^{n}}{\beta(n)^{2}}=$ 
$\sum_{n=0}^{\infty} \frac{\left(\overline{a_{0}} z\right)^{n}}{\beta(n)^{2}}=\overline{p(\mu \bar{z})}$ and $q(z)=\sum_{n=1}^{\infty} \frac{n\left(\bar{\mu} a_{0} z\right)^{n-1}}{\beta(n)^{2}}=\sum_{n=1}^{\infty} \frac{n\left(\overline{a_{0}} z\right)^{n-1}}{\beta(n)^{2}}=\overline{q(\mu \bar{z})}$ for every $z \in \mathbb{D}$. Hence, $\psi(z)=a_{2} p(z)=\overline{a_{2}} \overline{p(\mu \bar{z})}=\overline{\psi(\mu \bar{z})}$ for all $z \in \mathbb{D}$. Also, we have that for all $z, w \in \mathbb{D}$

$$
\begin{aligned}
K_{\mu \bar{w}}(\phi(z)) & =\sum_{n=0}^{\infty} \frac{(\bar{\mu} w \phi(z))^{n}}{\beta(n)^{2}} \\
& =\sum_{n=0}^{\infty} \frac{\left(a_{0} \bar{\mu} w+\bar{\mu} \beta(1)^{2} a_{1} w z \frac{q(z)}{p(z)}\right)^{n}}{\beta(n)^{2}} \quad \text { using eq.(2.1)) } \\
& =\sum_{n=0}^{\infty} \frac{\left(\overline{a_{0}} w+\bar{\mu} \beta(1)^{2} \overline{a_{1}} w z \frac{\overline{q(\mu \bar{z})}}{\overline{p(\mu \bar{z})}}\right)^{n}}{\beta(n)^{2}} \\
& =\sum_{n=0}^{\infty} \frac{(w \overline{\phi(\mu \bar{z})})^{n}}{\beta(n)^{2}} \\
& =K_{\bar{w}}(\overline{\phi(\mu \bar{z})}) .
\end{aligned}
$$

Thus, (3.1) holds true for every $z, w \in \mathbb{D}$ and we get that the weighted composition operator $W_{\psi, \phi}$ is Hermitian on $H^{2}(\beta)$.

The next theorem provides necessary and sufficient conditions under which a bounded and $\mathcal{J}_{\mu}$-symmetric operator $W_{\psi, \phi}$ is a coisometry.

Theorem 3.2. Let $W_{\psi, \phi}$ be a bounded and $\mathcal{J}_{\mu}$-symmetric weighted composition operator on $H^{2}(\beta)$ such that $\phi(0)=0$. Then $W_{\psi, \phi}$ is a coisometry if and only if $\psi(0)$ and $\phi^{\prime}(0)$ are unimodular.

Proof. For every $w, z \in \mathbb{D}$, we compute

$$
\begin{aligned}
W_{\psi, \phi} W_{\psi, \phi}^{*} K_{w}(z) & =W_{\psi, \phi}\left(\overline{\psi(w)} K_{\phi(w)}(z)\right) \\
& =\psi(z) \overline{\psi(w)} K_{\phi(w)}(\phi(z)) \\
& =\psi(z) \overline{\psi(w)} \sum_{n=0}^{\infty} \frac{(\overline{\phi(w)} \phi(z))^{n}}{\beta(n)^{2}}
\end{aligned}
$$

Since $W_{\psi, \phi}$ is $\mathcal{J}_{\mu}$-symmetric, Theorem 2.1 implies that $\phi(z)=a_{1} z$ and $\psi(z)=a_{2}$ where $a_{1}=\phi^{\prime}(0)$ and $a_{2}=\psi(0)$.

Also, $W_{\psi, \phi} W_{\psi, \phi}^{*}=I$. Thus, for all $w, z \in \mathbb{D}, W_{\psi, \phi} W_{\psi, \phi}^{*} K_{w}(z)=K_{w}(z)$ implies that

$$
\left|a_{2}\right|^{2}\left(1+\sum_{n=1}^{\infty} \frac{\left(\left|a_{1}\right|^{2} \bar{w} z\right)^{n}}{\beta(n)^{2}}\right)=1+\sum_{n=1}^{\infty} \frac{(\bar{w} z)^{n}}{\beta(n)^{2}}
$$

In particular, taking $w=z=0$ in (3.5), we obtain that $\left|a_{2}\right|=1$. Next, on differentiating (3.5) with respect to $z$, we get

$$
\left|a_{1}\right|^{2} \bar{w}\left(\frac{1}{\beta(1)^{2}}+\sum_{n=2}^{\infty} \frac{n\left(\left|a_{1}\right|^{2} \bar{w} z\right)^{n-1}}{\beta(n)^{2}}\right)=\bar{w}\left(\frac{1}{\beta(1)^{2}}+\sum_{n=2}^{\infty} \frac{n(\bar{w} z)^{n-1}}{\beta(n)^{2}}\right) .
$$

On putting $z=0$ in (3.6), we deduce that $\left|a_{1}\right|=1$. Conversely, if $\left|a_{1}\right|=1=\left|a_{2}\right|$, then the equation (3.5) holds true for every $w, z \in \mathbb{D}$. Hence, the operator $W_{\psi, \phi}$ is a coisometry. 
Lemma 3.3. Suppose that the operator $W_{\psi, \phi}$ is bounded and complex symmetric with respect to conjugation $\mathcal{J}_{\mu}$ on $H^{2}(\beta)$ such that $\phi(0)=0$. Then $W_{\psi, \phi}^{*} W_{\psi, \phi} K_{w}(z)=|\psi(0)|^{2} K_{\left|\phi^{\prime}(0)\right|{ }^{2} w}(z)$ for every $w$, $z \in \mathbb{D}$.

Proof. On taking $\phi(0)=0$ in (2.1), we get $\phi(z)=a_{1} z$ and $\psi(z)=a_{2}$. For every $w, z \in \mathbb{D}$, the expression $W_{\psi, \phi}^{*} W_{\psi, \phi} K_{w}(z)$ is evaluated as follows:

$$
\begin{aligned}
W_{\psi, \phi}^{*} W_{\psi, \phi} K_{w}(z) & =W_{\psi, \phi}^{*}\left(\psi(z) K_{w}(\phi(z))\right) \\
& =W_{\psi, \phi}^{*}\left(a_{2} \sum_{n=0}^{\infty} \frac{\left(a_{1} \bar{w} z\right)^{n}}{\beta(n)^{2}}\right) \\
& =a_{2} W_{\psi, \phi}^{*} K_{\overline{a_{1}} w}(z) \\
& =a_{2} \overline{\psi\left(\overline{a_{1}} w\right)} K_{\phi\left(\overline{a_{1}} w\right)}(z) \\
& =\left|a_{2}\right|^{2}\left(\sum_{n=0}^{\infty} \frac{\left(\left|a_{1}\right|^{2} \bar{w} z\right)^{n}}{\beta(n)^{2}}\right) .
\end{aligned}
$$

Hence, we obtain that $W_{\psi, \phi}^{*} W_{\psi, \phi} K_{w}(z)=|\psi(0)|^{2} K_{\left|\phi^{\prime}(0)\right|{ }^{2} w}(z)$.

In the following result, we have obtained the equivalence of isometry and coisometry of a bounded and $\mathcal{J}_{\mu}$-symmetric weighted composition operator on $H^{2}(\beta)$ under a certain condition.

Theorem 3.4. Let $W_{\psi, \phi}$ be a bounded and $\mathcal{J}_{\mu}$-symmetric weighted composition operator on $H^{2}(\beta)$ such that $\phi(0)=0$. Then $W_{\psi, \phi}$ is an isometry if and only if $W_{\psi, \phi}$ is a coisometry.

Proof. It can be obtained from Lemma 3.3 and equation (3.5) that for every $w, z \in \mathbb{D}, W_{\psi, \phi}$ is an isometry if and only if $W_{\psi, \phi}^{*} W_{\psi, \phi} K_{w}(z)=K_{w}(z)$ if and only if $\left|a_{2}\right|^{2}\left(\sum_{n=0}^{\infty} \frac{\left(\left|a_{1}\right|^{2} \bar{w} z\right)^{n}}{\beta(n)^{2}}\right)=\sum_{n=0}^{\infty} \frac{(\bar{w} z)^{n}}{\beta(n)^{2}}$ if and only if $W_{\psi, \phi} W_{\psi, \phi}^{*} K_{w}(z)=K_{w}(z)$.

Corollary 3.5. Let $W_{\psi, \phi}$ be a bounded and $\mathcal{J}_{\mu}$-symmetric weighted composition operator on $H^{2}(\beta)$ such that $\phi(0)=0$. Then $W_{\psi, \phi}$ is unitary if and only if $W_{\psi, \phi}$ is an isometry.

Acknowledgement: The authors are thankful to the reviewers for their valuable suggestions and comments.

\section{References}

[1] Cowen, C.C., MacCluer, B.D.: Composition operators on spaces of analytic functions. Studies in Advanced Mathematics, CRC Press, Boca Raton, FL, (1995). MR1397026

[2] Garcia, S.R., Hammond, C.: Which weighted composition operators are complex symmetric?. Concrete operators, spectral theory, operators in harmonic analysis and approximation, 171-179, Oper. Theory Adv. Appl., 236, Birkhäuser/Springer, Basel. MR3203059

[3] Garcia, S.R., Prodan, E., Putinar, M.: Mathematical and physical aspects of complex symmetric operators. J. Phys. A 47 , no. 35, 353001, 54 pp. (2014) MR3254868

[4] Garcia, S.R., Putinar, M.: Complex symmetric operators and applications. Trans. Amer. Math. Soc. 358, no. 3, 12851315 (2006). MR2187654 
[5] Garcia, S.R., Putinar, M.: Complex symmetric operators and applications. II. Trans. Amer. Math. Soc. 359, no. 8, 3913-3931 (2007). MR2302518

[6] Garcia, S.R., Wogen, W.R.: Some new classes of complex symmetric operators. Trans. Amer. Math. Soc. 362 , no. 11, 6065-6077 (2010). MR2661508

[7] Jung, S., Kim, Y., Ko, E., Lee, J.E.: Complex symmetric weighted composition operators on $H^{2}(\mathbb{D})$. J. Funct. Anal. 267 , no. 2, 323-351 (2014). MR3210031

[8] Lim, R., Khoi, L.H.: Complex symmetric weighted composition operators on $\mathcal{H}_{\gamma}(\mathbb{D})$. J. Math. Anal. Appl. 464, no. 1, 101-118 (2018). MR3794079

[9] Narayan, S.K., Sievewright, D., Thompson, D.: Complex symmetric composition operators on $H^{2}$. J. Math. Anal. Appl. 443 , no. 1, 625-630 (2016). MR3508506

[10] Shapiro, J.H.: Composition operators and classical function theory. Universitext: Tracts in Mathematics, SpringerVerlag, New York (1993). MR1237406 